\title{
Assessment of Bone Pain Response in Cancer Patients Receiving Single Dose of Sm-153 EDTMP Therapy
}

\section{Mai Elzahry ${ }^{1 *}$, Waleed Diab² and Helmut Sinzinger ${ }^{3}$}

${ }^{1}$ Faculty of Medicine, Department of Clinical Oncology and Nuclear Medicine, South Valley University, Qena 83523, Egypt ${ }^{2}$ Faculty of Medicine, Department of Clinical Oncology and Nuclear Medicine, Assiut University, Assiut, Egypt

3/SOTOPIX, Institute for Nuclear Medicine, Vienna, Austria

\begin{abstract}
Background: Painful metastatic bone dissemination is a common complication of solid malignant tumors that can lead to severe morbidity. There are different treatment strategies currently available for pain relief. Among those, we obtained clinical experience with Sm-153 EDTMP.
\end{abstract}

Objective: It was to evaluate the overall therapeutic response in 110 patients who underwent a single dose of Sm-153 EDTMP therapy and report if there is a significant correlation of metastatic bone pain response with gender, pathology of primary cancer, patient's age, extent of the metastatic bone dissemination.

Patients and methods: 110 cancer patients were included in this retrospective analysis, $63(57.2 \%)$ males (age range; 52-89 years) and 47 (42.7\%) females (age range: 35-84 y), their diagnosis were prostate and breast cancer, respectively. all patients performed conventional bone scintigraphy to prove the evidence of metastatic bone dissemination. Pain severity was assessed clinically and according to WHO Analgesia Scale.

Results: Out of 110 cancer patients received a therapeutic dose of $153 \mathrm{Sm}$ - EDTMP for palliation of painful metastatic bone lesions, 93.6\% (103/110) showed overall therapeutic response and $6.4 \%(7 / 110)$ showed no response at all. $61 \%(67 / 110)$ of patients were completely pain-free, $32.6 \%(36 / 110)$ were partially responded to therapy, that response shows insignificant relations with the patients gender, pathology of primary tumor, patients age as well as the extent of metastatic bone dissemination.

Conclusion: The results of this study showed that a single dose of Sm-153 EDTMP offers an effective treatment option in patients with painful metastatic bone disseminations irrespective to their gender, age, tumor pathology as well as to the bone lesions extent.

Keywords: Bone pain response; Cancer patients; Sm-153 EDTMP therapy

\section{Introduction}

Many patients with cancer develop symptomatic skeletal metastases at an advanced stage of their disease, they often complicated with pain [1]. Its prevalence is estimated to $73 \%$ in breast and about $68 \%$ in prostate cancer [2]. Over the past few decades, several radiopharmaceuticals have been developed with bone seeking properties that provide palliation of pain to multiple areas of skeleton without any significant hematological or soft tissue toxicity [3].

Sm-153 is the most widely used radioisotope for metastatic bone pain palliation for all lesions showing up in bone scintigraphy not only in patients with cancer prostate (osteoblastic metastases), but even in those presenting with a mixed pattern of osteolytic and osteoblastic metastases, like those seen in breast cancer [4].

\section{Nature of the Study}

This is a retrospective clinical study on 110 patients who underwent single dose of Sm-153 EDTMP for therapy of painful metastatic bone lesions.

Aim of the study was to evaluate the overall therapeutic response and report if there is a significant correlation of metastatic bone pain response with gender, pathology of primary cancer, patient's age as well as extent of the metastatic bone dissemination.

\section{Treatment Design}

Sm-153 EDTMP therapy was performed according to the Vienna protocol [5]. The protocol is defined as follows: A single dose of $30 \mathrm{mCi}$ (1.1 GBq) Sm-153 EDTMP was administered through a slow intravenous injection on an outpatient basis. Whole body bone Scintigraphy was performed usually on the next day, anyway, about 20 hours after radionuclide application to achieve complete blood clearance, using large field of view double headed $\gamma$-camera, LEHR-collimation, energy window $20 \%, 103 \mathrm{KeV}$, acquisition mode contionusely $15 \mathrm{~cm} / \mathrm{min}$, early images $(<4$ hours) showed significantly lower quality.

The study included patients had been treated at Department of Nuclear Medicine, Medical University of Vienna, Austria. All patients were followed up for 12 weeks, conventional bone scintigraphy (Tc99m MDP), analgesic consumption (according to WHO Analgesia Scale which based on analgesia requirements for each patient), pain symptoms, blood cell count were recorded by the patients and/or family members during each visit. The patient was allowed to adjust the dose of his/her medications if symptoms changed. The patients were asked to get the haemogram 3 and 6 weeks after therapy up to 12 weeks

*Corresponding author: Mai Elzahry, Faculty of Medicine, Department of Clinical Oncology and Nuclear Medicine, South Valley University, Qena 83523, Egypt, Tel: +2 01010720929; E-mail: mai.rifat@hotmail.com

Received November 17, 2017; Accepted November 20, 2017; Published November 27, 2017

Citation: Elzahry M, Diab W, Sinzinger H (2017) Assessment of Bone Pain Response in Cancer Patients Receiving Single Dose of Sm-153 EDTMP Therapy. J Nucl Med Radiat Ther 8: 349. doi: 10.4172/2155-9619.1000349

Copyright: ( 2017 Elzahry M, et al. This is an open-access article distributed under the terms of the Creative Commons Attribution License, which permits unrestricted use, distribution, and reproduction in any medium, provided the original author and source are credited. 
and provide the report to the physician telephonically. At the end of 12 weeks, the patient was asked to come to the clinic for subsequent detailed assessment and recording of data.

This study was approved by the Ethics Commission at the Medical University of Vienna and the Vienna General Hospital (AKH), each patient was explained the details of the procedure, benefits and side effects of therapy and the follow-up protocol and all patients provided written informed consent.

\section{Statistical Analysis}

Date entry and data analysis were done using SPSS version 16 (Statistical Package for Social Science). The data of the patients were retrospectively collected. Continuous variables were summarized as means and standard deviations, while categorical variables were summarized as numbers and percentage. "Independent-samples $\mathrm{T}$ test" was used to test for significance between two variables. For all $\mathrm{P}$-values $<0.05$ were selected as significant.

\section{Design and Methodology}

This retrospective analysis included 110 patients, 63 (57.2\%) males [age range; $52-89$ years] and $47(42.7 \%)$ females [age range: 35-84 years], their primary cancer were prostate and breast cancer, respectively. The majority of the patients have more than 10 metastatic bone lesions on conventional bone scintigraphy representing $71 \%$ of all patients (78/110) (Table 1).

Before initiating treatment, all patients performed conventional bone scintigraphy to prove the evidence of metastatic bone dissemination. Bone pain was significant in all patients Pain severity was assessed clinically and according to WHO Analgesia Scale. Pain severity was evaluated based on analgesia requirements according to WHO Analgesia Scale.

\section{Results}

A total of 110 cancer patients received a single dose of Sm-153 EDTMP therapy for palliation of painful bone metastases, $93.6 \%$ $(103 / 110)$ showed overall therapeutic response and $6.4 \%$ (7/110) showed only no response at all. $61 \%(67 / 110)$ of patients were completely painfree, $32.6 \%(36 / 110)$ were partially responded to therapy (Table 2$)$.

Pain response is assessed in all 110 patients after administration of the single dose of Sm-153 EDTMP depending on their gender (pathology of primary cancer), patient's age and the extent of metastatic bone lesions.

\section{Gender/pathology of primary cancer}

Breast cancer patients expressed high partial response to pain (40.4\%) as compared to prostate cancer $(27 \%)$, while prostate cancer patients expressed a high complete cure $(63.5 \%)$ as compared to

\begin{tabular}{|c|c|c|}
\hline Age (years) & Ca prostate & Ca breast \\
\hline Mean \pm SD & $71.1 \pm 8.3$ & $58.3 \pm 13.2$ \\
\hline Range & $52-89 \mathrm{y}$ & $35-84 \mathrm{y}$ \\
\hline \multicolumn{2}{|c|}{ Gender } \\
\hline Males & $63 / 57.3$ \\
\hline Females & 0 \\
\hline \multicolumn{2}{|c|}{ Extent of bone lesions (n/\%) } \\
\hline Less than 10 lesions & $20 / 31.7$ & $12 / 25.5$ \\
\hline More than 10 lesions & $43 / 68.3$ & $35 / 74.5$ \\
\hline All values expressed in number/percentage
\end{tabular}

Table 1: Demographic characterstics. breast cancer (57.4\%). Generally, the overall response to pain among breast cancer patients is higher $(97.8 \%)$ than that of prostate $(90.5 \%)$. Statistically there is no such difference among prostate and breast cancer patients in their response to pain (all P-values $>0.05$ ) as shown in Table 2 .

\section{Age}

Data in Table 3 describes the difference in pain response among the different age groups of prostate and breast cancer patients. The patients are divided according to age into 2 groups, the first group representing the patients who aged 70 years old or younger and the second group representing the patients who are older than 70 years old. In both age groups, the prostate cancer patients who were older than 70 years expressed high complete cure (71.8\%) than that of breast cancer patients (33.4\%), while the older breast cancer patients expressed a high partial response $(66.6 \%)$ to therapy as compared to prostate cancer patients at the same age group, $9.7 \%$ of prostate cancer patients expressed no response to therapy while the all breast cancer patients achieved overall response to therapy. Statistically, there is no significant difference in pain response among the both age groups of prostate and breast cancer patients (P-values $>0.05$ )

\section{Extent of bone metastases}

Cancer patients who have more than 10 bone lesions on bone scan expressed a high complete response (60.3\%) after the first dose of therapy as compared to those who have less than 10 bone lesions (50\%). Generally, the rate of the response among the cancer patients having more than 10 bone lesions shows an equal percentage (78.2\%) as compared to the other group of the patients who have less than 10 bone lesions $(78.1 \%)$ with no statistical difference in pain response between the both groups of patients ( $\mathrm{P}$-values $>0.05$ ).

\section{Discussion}

Radionuclide therapy has been gaining popularity $\mathrm{m}$ the management of painful osseous metastases. This form of palliative therapy has the advantages of targeting all the involved sites but limiting the dose to normal tissue [6]. Radionudide therapy was used in patients with widespread metastatic disease, who would not benefit much from other therapies [7].

There are several studies discussed the efficacy of Sm-153 EDTMP as a palliative radionuclide therapy for metastatic bone pain and

\begin{tabular}{|c|c|c|}
\hline Variables & Ca prostate & Ca breast \\
\hline Total n/\% & $63 / 57.3$ & $47 / 42.7$ \\
\hline \multicolumn{3}{|c|}{ Duration (12 weeks) (n/\%) } \\
\hline Complete response & $40 / 63.5$ & $27 / 57.4$ \\
\hline Partial response & $17 / 27$ & $19 / 40.4$ \\
\hline \multicolumn{2}{|c|}{ No response } & $6 / 9.5$ \\
\hline All values expressed in number/percentage \\
\hline
\end{tabular}

Table 2: Therapeutic response to Sm-153 EDTMP therapy.

\begin{tabular}{|l|c|c|c|c|}
\hline \multirow{2}{*}{ Variables } & \multicolumn{2}{|c|}{$\leq 70 \mathbf{y}$} & \multicolumn{2}{c|}{$>70 \mathrm{y}$} \\
\cline { 2 - 5 } & \multicolumn{2}{|c|}{$(\mathbf{n = 6 9 )}$} & \multicolumn{2}{c|}{$(\mathbf{n = 4 1 )}$} \\
\cline { 2 - 5 } & Ca prostate & Ca breast & Ca prostate & Ca breast \\
\hline Total $\mathrm{n} / \%$ & $(31 / 45)$ & $(38 / 55)$ & $(32 / 78.1)$ & $(9 / 21.9)$ \\
\hline Complete response & $17 / 54.8$ & $25 / 65.8$ & $23 / 71.8$ & $3 / 33.4$ \\
\hline Partial response & $11 / 35.5$ & $12 / 31.6$ & $6 / 18.7$ & $6 / 66.6$ \\
\hline No response & $3 / 9.7$ & $1 / 2.6$ & $3 / 9.5$ & 0 \\
\hline All values expressed in number/percentage \\
\hline
\end{tabular}

Table 3: Sm-153 EDTMP therapy induced pain response vs. patient's age. 


\begin{tabular}{|c|c|c|c|c|}
\hline \multirow{3}{*}{ Variables } & \multicolumn{2}{|c|}{$\leq 10$ bone lesions } & \multicolumn{2}{|c|}{$>10$ bone lesions } \\
\hline & \multicolumn{2}{|c|}{$(n=32)$} & \multicolumn{2}{|c|}{$(n=78)$} \\
\hline & Ca prostate & Ca breast & Ca prostate & Ca breast \\
\hline$(\mathrm{n} / \%)$ & $(20 / 62.5)$ & $(12 / 37.5)$ & $(43 / 55)$ & $(35 / 45)$ \\
\hline Complete response & $12 / 60$ & $4 / 33.4$ & $30 / 69.7$ & $17 / 48.6$ \\
\hline Partial response & $5 / 25$ & $4 / 33.3$ & $4 / 9.3$ & $10 / 28.6$ \\
\hline No response & $3 / 15$ & $4 / 33.3$ & $9 / 21$ & $8 / 22.8$ \\
\hline
\end{tabular}

Table 4: Sm-153 EDTMP therapy induced pain response vs. extent of bone metastases.

\begin{tabular}{|c|c|c|c|c|}
\hline Name of Author & Cases (n) and diagnosis & Response(R) & Percentage(\%) & Duration(T) \\
\hline Wang RF & 9 with confirmed malignancies & overall $\mathrm{R}$ & 77.8 & $>3$ weeks \\
\hline Dolezal J & 43 females with breast cancer & overall $\mathrm{R}$ & 72 & 3 months \\
\hline Gonzalez CL & 277 prostate, breast and others & overall $\mathrm{R}$ & 54 & $>3$ weeks \\
\hline Coronado M & 28 breast, 27 prostate & complete R & 21 & $3 \mathrm{~m}$ \\
\hline Lakovou I & 36 female with breast cancer & complete R & 52 & 16 weeks \\
\hline Tripathi M & 86 from various primaries & overall $\mathrm{R}$ & 73 & $>16$ weeks \\
\hline Serafini AN & 118 from various malignancies & overall R & 43 & During 16 weeks \\
\hline Vina JC & 94 prostate, breast and others & overall $\mathrm{R}$ & 85 & ( 7 days -3 months) \\
\hline Beiki D & 16 breast, prostate, thyroid and paraganglioma & complete R & 75 & At $8^{\text {th }}$ week \\
\hline
\end{tabular}

Table 5: Overview of relevant studies with Sm-153 EDTMP.

calculation of the overall or/and complete response rate of bone pain in relation to the time of therapy as shown in Table 4. Previous relevant studies (Table 5), reported a great variation of pain response rate in relation to the time of therapy among cancer patients with different confirmed primaries. For example, Vina et al. reported a high overall therapeutic response $85 \%$ of 94 patients over 3 months [8], this response rate is slightly higher as compared with Wang RF study (77.8\% after 3 weeks) [9]. The other two studies of González et al. and Tripathi et al. reported $75 \%$ vs. $73 \%$ within 4 months) [10,11], while Serafini et al. and Lakovou et al. registered a low response rate to pain in (43 vs. 52\%, respectively) through 16 weeks $[12,13]$.

In the present study, overall response rate was $93.6 \%$ within the time of follow up ( 3 months after the first dose of Sm-153 EDTMP). As compared with the other relevant studies discussed in Table 5 , this rate of response considered fairly high as compared to Serafini et al. study who achieved only $43 \%$ of pain response during the same duration of time, taking into consideration that the number of our patients was nearly similar to his population (118 and 110) respectively [12].

Complete responders in this study were $67 / 110(61 \%)$ while $36 / 110$ (32.6\%) of patients showed partial pain response and only 7/110 (6.4\%) of patients expressed no response to therapy. This compared with Dolezal et al. study which showed $42 \%$ of patients expressed complete response, $30 \%$ partial response and $28 \%$ non-responders [14], while at Lakovou et al. study, pain palliation was complete in $52 \%$ of the patients, partial in $31 \%$ and absent in $16 \%$ [13].

Coronado M. study recorded a very low rate of complete response to therapy $(21 \%)$, partial response $(40 \%)$ with $(24 \%)$ of patients showed absent response to therapy [15]. After injection of 153Sm-EDTMP, response was recognized in $90.3 \%$ prostate cancer and $97.9 \%$ of breast cancer patients, a total of $7 / 110$ prostate and breast cancer patients did not respond to therapy. As compared with other studies showed 40 to $85.5 \%$ response rates in cancer breast and 70 to $80 \%$ response rate in cancer prostate [16-18], we reported insignificant difference in therapeutic response rate between prostate and breast cancer $(90.5 \%$ and $97.8 \%$ respectively), this finding also recorded by Tripathi et al. as well as Baczyk et al. who did not show any significant difference in response rate among prostate and breast cancer patients $(80.6 \%$ and $80.4 \%)$ respectively $[11,19]$.

Statistically, there is no difference in therapeutic response among different age groups of the patients, $94.2 \%$ of responders was younger than 70 years and $92.6 \%$ of responders older than 70 years while $5.8 \%$ of non-responders was younger than 70 years and $7.4 \%$ of non-responders older than 70 years which is also compatible with the findings of Beiki et al. and Sinzinger et al. [5,20]. A comparative analysis also was designed to identify the factors related to therapeutic response between responders and non-responders by Tian. The mean and range of age in the groups were $54 \pm 10.9$ years (27-72 years) and $57 \pm 11.1$ years ( 30 82 years), respectively. However, statistically, there was no difference between the two groups [21].

\section{Study limitations}

1. Disadvantage of this study is that it is a retrospective one, that data collection was limited because a number of patients died.

2. Advantage is that it was conducted on a fairly high number of patients followed of a single site with a quite extensive work-up program.

\section{Conclusion}

The results of this study showed that a single dose of $153 \mathrm{Sm}$ EDTMP offers an effective treatment option in patients with painful metastatic bone disseminations irrespective to their gender, age, primary tumor pathology as well as to the bone lesions extent. Overall, $61 \%$ of cancer patients were completely pain free after a single dose of Sm-153 EDTMP, 33\% partially responded and less than 10\% showed no response. 
Citation: Elzahry M, Diab W, Sinzinger H (2017) Assessment of Bone Pain Response in Cancer Patients Receiving Single Dose of Sm-153 EDTMP Therapy. J Nucl Med Radiat Ther 8: 349. doi: 10.4172/2155-9619.1000349

\section{References}

1. Lam MG, Klerk De JM, Rijk Van PP, Zonnenberg BA (2007) Bone seeking radiopharmaceuticals for palliation of pain in cancer patients with osseous metastases. Anticancer Agents Med Chem 7: 381-397.

2. Galasko C (1981) The anatomy and pathways of skeletal metastases. In: Weiss L, Gilbert A (eds). Bone metastases. Boston, GK Hall.

3. Michael T (2012) The role of bone seeking radionuclides in the palliative treatment of patients with painful osteoblastic skeletal metastases. Cancer Control 19: 137-144.

4. Menda Y, Bushnell DL, Williams RD, Miller S, Thomas MO (2000) Efficacy and safety of repeated ${ }^{153} \mathrm{Sm}$-Lexidronam treatment in a patient with prostate cancer and metastatic bone pain. Clin Nucl Med 25: 698-700.

5. Sinzinger H, Weiss K, Hiltunen J (2009) Background, reasons and benefits using the Vienna Protocol for the treatment of painful bone recurrences with ${ }^{153}$ Sm-EDTMP. Anticancer Res 29: 3393-3396.

6. Robinson RG, Preston DF, Schiefelbein M, Baxter KG ( 1995) Strontium-89 therapy for the palliation of pain due to osseous metastases. JAMA 274 420-424.

7. Papatheofanis FJ (1995) Variation in oncalogic opinion regarding management of metastatic bone pain with systemic radionuclide therapy. J Nud Med 40: $1420-1423$.

8. Vina JC (2005) Metastasic bone pain management with radioactive isotopes. Braz Arch Biol Techn 48: 127-135.

9. Wang RF, Zhang CL, Zhu SL, Zhu M (2003) A comparative study of 153Samarium-ethylenediaminetetramethylene phosphonic acid with pamidronate disodium in the treatment of patients with painful metastatic bone cancer. Med Princ Pract 12: 97-101.

10. Gonzalez LC, Murphy AC, Romero PP, Lopez MP, Garcia CM, et al. (2014) $153 S m-E D T M P$ for pain relief of bone metastases from prostate and breas cancer and other malignancies. Arch Med Res 45: 301-308.

11. Tripathi M, Singhal T, Chandrasekhar N, Kumar P, Bal C, et al. (2006) Samarium-153 ethylenediaminetetramethylene phosphonate therapy for bone pain palliation in skeletal metastases. Indian J Cancer 43: 86-92.
12. Serafini AN, Houston SJ, Resch I, Quick DP, Grund FM, et al. (1998) Palliation of pain associated with metastatic bone cancer using samarium -153 Lexidronam: A double-blind: A double blind placebo controlled clinicak trial. J Clin Oncol 16: 1574-1581.

13. Lakovou I, Doumas A, Badiavas K, Mpalaris V, Frangos S, et al. (2009) Pain palliative therapy in women with breast cancer osseous metastatic disease and the role of specific serum cytokines as prognostic factors. Cancer Biother Radiopharm 3: 116-123.

14. Dolezal J, Vizda J, Cermakova E (2003) Myelotoxicity after systemic radionuclide therapy of painful bone metastases with 153Samarium-EDTMP. Vnitr Lek 49: 189-193.

15. Coronado M, Redondo A, Coya J, Espinosa E, Couto RM, et al. (2006) Clinica role of Sm-EDTMP in the treatment of painful bone metastatic disease. Clin Nucl Med 31: 605-610.

16. Collins C, Eary JF, Donaldson G, Vernon C, Bush NE, et al. (1993) Samarium153-EDTMP in bone metastases of hormone refractory prostate carcinoma: A phase I/II trial. J Nucl Med 34: 1839-1844.

17. Resche I, Chatal JF, Pecking A, Ell P, Duchesne G, et al. (1997) A dosecontrolled study of $153 \mathrm{Sm}$ ethylene-diamine-tetramethylene- phosphonate (EDTMP) in the treatment of patients with painful bone metastases. Eur Cancer 33: 1583-1591.

18. Turner JH, Martindale AA, Sorby P, Hetherington EL, Fleay RF, et al. (1989) Samarium-153 EDTMP therapy of disseminated skeletal metastasis. Eur J Nuc Med 15: 784-795.

9. Baczyk M, Czepczyński R, Milecki P, Pisarek M, Oleksa R, et al. (2007) 89S versus 153Sm-EDTMP: Comparison of treatment efficacy of painful bone metastases in prostate and breast carcinoma. Nucl Med Commun 28: 245-250.

20. Beiki D, Haddad P, Fallahi B, Keyvan A, Gholamrezanezhad A, et al. (2013) Effectiveness and complications of $153 \mathrm{Sm}$-EDTMP in palliative treatment of diffuse skeletal metastases. Iran J Nucl Med 21: 26-32.

21. Tian JH, Zhang JM, Hou QT, Oyang QH, Wang JM, et al. (1999) Multicentre trial on the efficacy and toxicity of single-dose samarium-153-ethylene diamine tetramethylene phosphonate as a palliative treatment for painful skeletal metastases in China. Eur J Nucl Med 26: 2-7. 